\title{
Meta-Analysis of Test Accuracy Studies with Multiple and Missing Thresholds: A Multivariate-Normal Model
}

Richard D Riley ${ }^{*}$, Yemisi Takwoingi ${ }^{1}$, Thomas Trikalinos ${ }^{2}$, Apratim Guha ${ }^{3}$, Atanu Biswas ${ }^{4}$, Joie Ensor ${ }^{1}$, R Katie Morris ${ }^{5,6}$ and Jonathan $\mathbf{J}$ Deeks ${ }^{1}$

${ }^{1}$ School of Health and Population Sciences, Public Health Building, University of Birmingham, Edgbaston, Birmingham, B15 2TT, UK

${ }^{2}$ Center for Evidence-based Medicine, Center for Statistical Sciences, and Department of Health Services, Policy \& Practice, Brown University School of Public Health, Brown University, Providence, RI 02912, USA

${ }^{3}$ Production and Quantitative Methods Area, Indian Institute of Management, Ahmedabad-380015, India

${ }^{4}$ Applied Statistics Unit, Indian Statistical Institute, 203 B.T. Road, Kolkata-700 108, India

${ }^{5}$ Centre for Women's \& Children Health and the School of Clinical and Experimental Medicine, College of Medical and Dental Sciences; University of Birmingham, Birmingham, B15 2TT, UK

${ }^{6}$ Fetal Medicine Centre, Birmingham Women's Hospital NHS Foundation Trust, Birmingham, UK

\begin{abstract}
Background: When meta-analysing studies examining the diagnostic/predictive accuracy of classifications based on a continuous test, each study may provide results for one or more thresholds, which can vary across studies. Researchers typically meta-analyse each threshold independently. We consider a multivariate meta-analysis to synthesise results for all thresholds simultaneously and account for their correlation.
\end{abstract}

Methods: We assume that the logit sensitivity and logit specificity estimates follow a multivariate-normal distribution within studies. We model the true logit sensitivity (logit specificity) as monotonically decreasing (increasing) functions of the continuous threshold. This produces a summary ROC curve, a summary estimate of sensitivity and specificity for each threshold, and reveals the heterogeneity in test accuracy across studies. Application is made to 13 studies of protein:creatinine ratio $(P C R)$ for detecting significant proteinuria in pregnancy that each report up to nine thresholds, with 23 distinct thresholds across studies.

Results: In the example there were large within-study and between-study correlations, which were accounted for by the method. A cubic relationship on the logit scale was a better fit for the summary ROC curve than a linear or quadratic one. Between-study heterogeneity was substantial. Based on the summary ROC curve, a PCR value of 0.30 to 0.35 corresponded to maximal pair of summary sensitivity and specificity. Limitations of the proposed model include the need to posit parametric functions for the relationship of sensitivity and specificity with the threshold, to ensure correct ordering of summary threshold results, and the multivariate-normal approximation to the within-study sampling distribution.

Conclusion: The joint analysis of test performance data reported over multiple thresholds is feasible. The proposed approach handles different sets of available thresholds per study, and produces a summary ROC curve and summary results for each threshold to inform decision-making.

Keywords: Multivariate meta-analysis; Test accuracy; Diagnostic test; Summary ROC curve; Correlation; Sensitivity; Specificity

\section{Introduction}

In the evaluation of diagnostic or predictive tests, meta-analysis methods are needed to synthesise the evidence about test accuracy from multiple studies. Most meta-analysis methods proposed in the literature consider a single two by two table from each study, which provides the number of true positives, true negatives, false positives, and false negatives [1-4]. However, when the test is measured on a continuous scale studies often report test performance at multiple thresholds, each relating to a different choice of threshold above which test results are classed as 'positive' and below which test results are classed as 'negative', either by reporting multiple two by two tables or labelled ROC curves. In this situation, researchers may select single thresholds per study, to allow estimation of a summary ROC curve using standard metaanalysis approaches [1-8]; or they may do a separate meta-analysis for each reported threshold [9].

However, a common problem is that most studies do not report the same set of thresholds. For example, in an evaluation of the spot protein:creatinine ratio (PCR) for detecting significant proteinuria in pregnancy, Morris et al. extracted tables for 23 different thresholds across 13 studies; eight of the thresholds were considered by just one study, but the other 15 thresholds were considered in two or more studies (Table 1), with a maximum of six studies for any threshold [10]. In such situations, an approach that considers meta-analysis for each threshold independently will omit any studies that do not report the threshold of interest, and thus also ignore information from other thresholds that are available in those studies. In particular, test accuracy results from neighbouring thresholds are likely to be quite similar, and results for a missing threshold are bounded between any pair of higher and lower thresholds that are available. Statistically it is appealing to utilise such related information and, if possible, 'borrow strength' by considering all thresholds simultaneously [11-14].

*Corresponding author: Richard D. Riley, School of Health and Population Sciences, Public Health Building, University of Birmingham, Edgbaston, Birmingham, B15 2TT, UK, Tel: 0121414 7508; Fax: 0121414 7878; E-mail: r.d.riley@bham.ac.uk

Received April 04, 2014; Accepted May 15, 2014; Published May 20, 2014

Citation: Riley RD, Takwoingi Y, Trikalinos T, Guha A, Biswas A, et al. (2014) Meta-Analysis of Test Accuracy Studies with Multiple and Missing Thresholds: A Multivariate-Normal Model. J Biomet Biostat 5: 196. doi:10.4172/21556180.1000196

Copyright: (C 2014 Riley RD, et al. This is an open-access article distributed under the terms of the Creative Commons Attribution License, which permits unrestricted use, distribution, and reproduction in any medium, provided the original author and source are credited. 
Citation: Riley RD, Takwoingi Y, Trikalinos T, Guha A, Biswas A, et al. (2014) Meta-Analysis of Test Accuracy Studies with Multiple and Missing Thresholds: A Multivariate-Normal Model. J Biomet Biostat 5: 196. doi:10.4172/2155-6180.1000196

Page 2 of 12

\begin{tabular}{|c|c|c|c|c|c|c|c|c|c|}
\hline First Author & threshold ID, $t$ & threshold value, $x$ & TP & FP & FN & TN & Total & High proteinuria & Normal proteinuria \\
\hline \multirow[t]{5}{*}{ Al Ragib } & 1 & 0.13 & 35 & 51 & 4 & 95 & 185 & 39 & 146 \\
\hline & 6 & 0.18 & 33 & 42 & 6 & 104 & & & \\
\hline & 7 & 0.19 & 33 & 39 & 6 & 107 & & & \\
\hline & 8 & 0.2 & 31 & 38 & 8 & 108 & & & \\
\hline & 22 & 0.49 & 29 & 23 & 10 & 123 & & & \\
\hline \multirow[t]{6}{*}{ Durnwald } & 3 & 0.15 & 156 & 35 & 12 & 17 & & & \\
\hline & 8 & 0.2 & 152 & 27 & 16 & 25 & & & \\
\hline & 15 & 0.3 & 136 & 23 & 32 & 29 & 220 & 168 & 52 \\
\hline & 19 & 0.39 & 123 & 14 & 45 & 38 & & & \\
\hline & 20 & 0.4 & 120 & 12 & 48 & 40 & & & \\
\hline & 23 & 0.5 & 106 & 9 & 62 & 43 & & & \\
\hline \multirow[t]{6}{*}{ Dwyer } & 3 & 0.15 & 54 & 28 & 2 & 32 & 116 & 56 & 60 \\
\hline & 5 & 0.17 & 51 & 25 & 5 & 35 & & & \\
\hline & 7 & 0.19 & 50 & 18 & 6 & 42 & & & \\
\hline & 12 & 0.24 & 41 & 8 & 15 & 52 & & & \\
\hline & 14 & 0.28 & 37 & 3 & 19 & 57 & & & \\
\hline & 19 & 0.39 & 31 & 0 & 25 & 60 & & & \\
\hline Leonas & 15 & 0.3 & 277 & 7 & 5 & 638 & 927 & 282 & 645 \\
\hline Ramos & 23 & 0.5 & 25 & 1 & 1 & 20 & 47 & 26 & 21 \\
\hline Robert & 15 & 0.3 & 27 & 4 & 2 & 38 & 71 & 29 & 42 \\
\hline \multirow[t]{8}{*}{ Rodriguez } & 2 & 0.14 & 69 & 34 & 0 & 35 & 138 & 69 & 69 \\
\hline & 3 & 0.15 & 68 & 34 & 1 & 35 & & & \\
\hline & 4 & 0.16 & 68 & 26 & 1 & 43 & & & \\
\hline & 5 & 0.17 & 65 & 25 & 4 & 44 & & & \\
\hline & 6 & 0.18 & 62 & 24 & 7 & 45 & & & \\
\hline & 7 & 0.19 & 62 & 21 & 7 & 48 & & & \\
\hline & 8 & 0.2 & 60 & 19 & 9 & 50 & & & \\
\hline & 9 & 0.21 & 60 & 17 & 9 & 52 & & & \\
\hline \multirow[t]{6}{*}{ Saudan } & 8 & 0.2 & 14 & 27 & 0 & 59 & 100 & 14 & 86 \\
\hline & 13 & 0.25 & 13 & 14 & 1 & 72 & & & \\
\hline & 15 & 0.3 & 13 & 7 & 1 & 79 & & & \\
\hline & 18 & 0.35 & 12 & 4 & 2 & 82 & & & \\
\hline & 20 & 0.4 & 11 & 3 & 3 & 83 & & & \\
\hline & 21 & 0.45 & 10 & 0 & 4 & 86 & & & \\
\hline \multirow[t]{2}{*}{ Schubert } & 3 & 0.15 & 9 & 3 & 0 & 3 & 15 & 9 & 6 \\
\hline & 4 & 0.16 & 9 & 2 & 0 & 4 & & & \\
\hline Shahbazian & 8 & 0.2 & 35 & 2 & 3 & 41 & 81 & 38 & 43 \\
\hline \multirow[t]{6}{*}{ Taherian } & 2 & 0.14 & 67 & 7 & 6 & 20 & 100 & 73 & 27 \\
\hline & 3 & 0.15 & 67 & 3 & 6 & 24 & & & \\
\hline & 4 & 0.16 & 65 & 1 & 8 & 26 & & & \\
\hline & 5 & 0.17 & 64 & 1 & 9 & 26 & & & \\
\hline & 6 & 0.18 & 63 & 0 & 10 & 27 & & & \\
\hline & 8 & 0.2 & 59 & 0 & 14 & 27 & & & \\
\hline Wheeler & 9 & 0.21 & 59 & 13 & 9 & 45 & 126 & 68 & 58 \\
\hline \multirow[t]{9}{*}{ Yamasmit } & 7 & 0.19 & 29 & 6 & 0 & 7 & 42 & 29 & 13 \\
\hline & 9 & 0.21 & 29 & 5 & 0 & 8 & & & \\
\hline & 10 & 0.22 & 29 & 4 & 0 & 9 & & & \\
\hline & 11 & 0.23 & 28 & 3 & 1 & 10 & & & \\
\hline & 12 & 0.24 & 28 & 2 & 1 & 11 & & & \\
\hline & 13 & 0.25 & 28 & 1 & 1 & 12 & & & \\
\hline & 14 & 0.28 & 27 & 1 & 2 & 12 & & & \\
\hline & 16 & 0.31 & 26 & 1 & 3 & 12 & & & \\
\hline & 17 & 0.32 & 25 & 1 & 4 & 12 & & & \\
\hline
\end{tabular}

Table 1: PCR results for each threshold in each of the 13 studies of Morris et al. [10]. 
In this article we propose a new approach for meta-analysing diagnostic test accuracy studies when there are multiple threshold results per study, when the studies use the same (or similarly validated or standardised) methods of measuring the continuous test (e.g. blood pressure or a continuous biomarker, like prostate-specific antigen or proteinuria). Hamza et al. [14] proposed a multivariate random-effect meta-analysis approach and applied it when all studies report all of the thresholds of interest. However, we consider approaches that allow different sets of available thresholds per study and can accommodate studies that only provide one threshold. The Hamza approach models the (linear) relationship between threshold value and test accuracy within each study, but this is not possible in studies that only report one threshold. The Hamza method is also prone to convergence problems, prompting Putter et al. to propose an alternative survival model framework for meta-analysing the multiple thresholds [15]. However this too requires the multiple thresholds to be available in all studies. Others have also considered the multiple threshold issue [13,16-20]. Here we extend the bivariate-normal meta-analysis model proposed by Reitsma et al. [1], which originally only allowed one threshold result per study. We propose a model that accounts for within-study correlations in the sensitivities and specificities at various thresholds (induced because estimates for different thresholds are from the same patients), and allows for relationships between test performance metrics at the between-study level (induced because studies can use different sets of thresholds). The approach incorporates all reported thresholds, even those reported in just one study, and can include all available studies, even if they provide just one threshold. It leads to a summary ROC curve, and summary sensitivity and specificity results for each threshold.

The article is structured as follows. In section 2 we describe the motivating PCR dataset. In Section 3 we introduce the model and its possible specifications, describe the estimation process, obtain a summary ROC curve, and summarise test performance at each threshold. In Section 4 we apply the methods to the PCR data, and Section 5 concludes with some discussion.

\section{Motivating Example: Identification of Significant Proteinuria in Patients with Suspected Pre-eclampsia}

Pre-eclampsia is a major cause of maternal and perinatal morbidity and mortality, and occurs in 2-8\% of all pregnancies [21-24]. The diagnosis of pre-eclampsia is determined by the presence of elevated blood pressure combined with significant proteinuria ( $\geq 0.3 \mathrm{~g}$ per 24 hour) after the $20^{\text {th }}$ week of gestation in a previously normotensive, non-proteinuric patient [25]. The gold-standard method for detection of significant proteinuria is the 24 hour urine collection, but this is cumbersome, time consuming and inconvenient, to patients as well as hospital staff. A rapid and accurate diagnostic test for significant proteinuria is needed for timely decision-making.

The spot PCR has been shown to be strongly correlated with 24 hour protein excretion, and thus is a potential diagnostic test for significant proteinuria. Morris et al. [10] performed a systematic review and meta-analysis to assess the diagnostic accuracy of PCR for the detection of significant proteinuria in patients with suspected pre-eclampsia. Thirteen relevant studies were identified, and in each study the reference standard was proteinuria greater than or equal to $300 \mathrm{mg}$ in urine over 24 hours. Across the 13 studies, 23 different threshold values were considered for PCR, ranging from 0.13 to 0.50 (as this is a ratio, there are no units). Five studies provided diagnostic accuracy results (i.e. a 2 by 2 table showing the number of true positives, false positives, false negatives, and true negatives) for just one threshold, but the other eight studies reported results for each of multiple thresholds, up to a maximum of nine thresholds (Yamasmit study). Eight of the 23 thresholds were considered by just one study, but the other 15 thresholds were considered in two or more studies, up to a maximum of six studies (for threshold 0.20). The studies and thresholds are summarised in Table 1.

Meta-analysis is important here to summarise the diagnostic accuracy of PCR at each threshold from all the published evidence, to help ascertain whether PCR is a useful diagnostic test and to give insight into clinically useful thresholds.

\section{Methods}

To meta-analyse diagnostic test studies with multiple thresholds, we consider a multivariate meta-analysis model that extends the bivariate model proposed by Reitsma et al. [1]. Multivariate normality of the logit sensitivity and logit specificity is assumed for the within-study sampling distribution, which is an approximation to the exact multinomial distribution [14]. However, multivariate normal distributions with randomeffects are easier to fit than multinomial models and more readily available in standard software packages such as Stata [26]. They enable the correlation between thresholds to be estimated and utilised. This is crucial as at the within-study level the same patients are contributing to results at each threshold and this causes the multiple logit sensitivity estimates to be correlated across thresholds, and similarly the multiple logit specificity estimates to be correlated. Across studies, there is also potential between-study correlation in the true logit sensitivity and true logit specificity values. This multivariate-normal framework utilises all these correlations in the meta-analysis [27], and enables the joint, rather than separate, synthesis of multiple thresholds. The approach is a two-step process, with the second stage producing the summary meta-analysis results for each threshold and a summary ROC curve, if desired.

\section{Step 1: Estimating threshold results and their variance-covariance matrix in each study}

Let there be $i=1$ to $m$ studies that measure a continuous test result on $n_{1 i}$ diseased patients and $n_{0 i}$ non-diseased patients, whose true disease status is provided by a reference standard. In each study, at a particular threshold value, $x$, each patient's measured test value is classed as either 'positive' $(\geq x)$ or 'negative' $(<x)$. Then summarising test results over all patients produces aggregate data in the form of $r_{11 i x}$, the number of truly diseased patients in study $i$ with a positive test result at threshold $x$, and $r_{00 i x}$, the number of non-diseased patients in study $i$ with a negative test result. The observed sensitivity at threshold value of $x$ in each study is thus simply $r_{11 i x} / n_{1 i}$ and the observed specificity is $r_{00 i x} / n_{0 i}$; a logit transformation can then be applied to obtain logit sensitivity and logit specificity estimates at each threshold. If sensitivity or specificity is 0 or 1 , then to enable logit estimates a continuity correction of 0.5 could be added to each $r_{11 i x}$ and $r_{00 i x}$, and 1 added to each $n_{1 i}$ and $n_{0 i}$. We focus here only on the logit transformation, as it is the most common one used in diagnostic test meta-analysis, but Chu et al. highlight that others (e.g. log-log or probit) can also be used [28]. 
To obtain the within-study variance-covariance matrix for the logit estimates, containing their variances and correlations, one can maximise the multinomial likelihood for each study, written as a function of the probabilities that test values fall between particular thresholds. The process is now described. First, in each study order the available thresholds. For example, in the Al Ragib study (Table 1) the threshold values of $x=0.13,0.18$, $0.19,0.2$, and 0.49 could be considered $t_{i}=1,2,3,4$, and 5 respectively for this study, with $\max \left(t_{i}\right)=5$ (i.e. the total number of thresholds in study $i$ ). Then for the diseased people define probabilities, $p_{1 i S_{i}}$, where $S_{i}=1$ to $\max \left(t_{i}\right)$ and: $p_{1 i 1}$ is the probability a patient's test value falls below threshold $1, p_{1 i 2}$ is the probability a patient's test value falls between threshold 1 and 2 , and so on, with the constraint that $1-\sum_{S_{i}=1}^{\max \left(t_{i}\right)} p_{1 i S_{i}}$ is the probability a patient's test value falls above threshold $\max \left(t_{i}\right)$. Similarly define $\max \left(t_{i}\right)$ probabilities for non-diseased people, termed $P_{0 i S_{i}}$. Then write the multinomial likelihood in terms of these probabilities for diseased patients, $l_{i}(1)$, and non-diseased patients, $l_{i}(0)$.

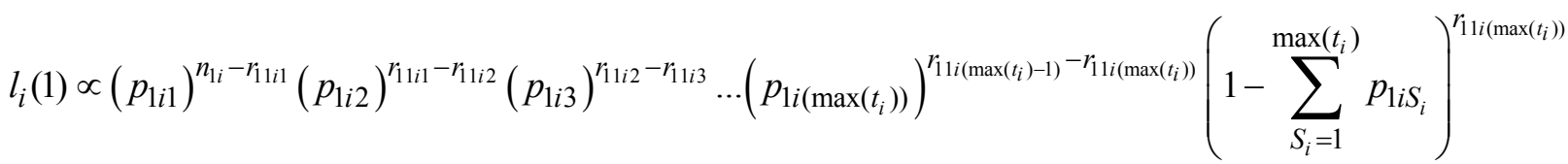

$$
\begin{aligned}
& l_{i}(0) \propto\left(p_{0 i 1}\right)^{r_{00 i 1}}\left(p_{0 i 2}\right)^{r_{00 i 2}-r_{00 i 1}}\left(p_{0 i 3}\right)^{r_{00 i 3}-r_{00 i 2}} \ldots\left(p_{0 i\left(\max \left(t_{i}\right)\right)}\right)^{r_{00 i\left(\max \left(t_{i}\right)\right)}-r_{00 i\left(\max \left(t_{i}\right)-1\right)}}\left(\left(1-\sum_{S_{i}=1}^{\max \left(t_{i}\right)} p_{0 i S_{i}}\right)\right)^{n_{0 i}-r_{00 i\left(\max \left(t_{i}\right)\right)}}
\end{aligned}
$$

and use statistical software (e.g. SAS Proc NLMIXED [29] or Stata 'maximize' [30]) to maximise the log likelihoods and subsequently obtain estimates of the logit sensitivity at threshold $t_{i}$

$$
y_{1 i t_{i}}=\operatorname{logit}\left(1-\sum_{S_{i}=1}^{t_{i}} \hat{p}_{1 i S_{i}}\right)
$$

and the logit specificity at threshold $t_{i}$

$$
y_{0 i t_{i}}=\operatorname{logit}\left(\sum_{S_{i}=1}^{t_{i}} \hat{p}_{0 i S_{i}}\right)
$$

and their variance-covariance matrix, for example using the delta method $[31,32]$. Example SAS code is available on request from the first author. For example, for the Al Ragib study (Table 1) the likelihoods are:

$$
\begin{aligned}
& l_{i}(1) \propto\left(p_{1 i 1}\right)^{39-35}\left(p_{1 i 2}\right)^{35-33}\left(p_{1 i 3}\right)^{33-33}\left(p_{1 i 4}\right)^{33-31}\left(p_{1 i 5}\right)^{31-29}\left(1-\sum_{S_{i}=1}^{5} p_{1 i S_{i}}\right)^{29} \\
& l_{i}(0) \propto\left(p_{0 i 1}\right)^{95}\left(p_{0 i 2}\right)^{104-95}\left(p_{0 i 3}\right)^{107-104}\left(p_{0 i 4}\right)^{108-107}\left(p_{0 i 5}\right)^{123-108}\left(1-\sum_{S_{i}=1}^{5} p_{0 i S_{i}}\right)^{146-123}
\end{aligned}
$$

One could also re-write $l_{i}(1)$ and $l_{i}(0)$ by expressing the probabilities in terms of the logit sensitivities and logit specificities [14], to directly estimate the logits and their variance-covariance matrix. Estimation of the likelihoods provides the within-study variances, $s_{1 i t_{i}}^{2}$ and $s_{0 i t_{i}}^{2}$, for the logit sensitivity and logit specificity estimates $y_{1 i t_{*}}$ and $y_{0 i t_{*}}$, respectively, and crucially also the within-study covariance between logit sensitivity estimates for each pair of thresholds (e.g. $\operatorname{cov}_{1 i\left(1, t_{i}\right)}$ is the covariance between $y_{1 i 1}$ and $y_{1 i t_{i}}$ ), and similarly the within-study covariance between logit specificity estimates for each pair of thresholds (e.g. $\operatorname{cov}_{0 i\left(1, t_{\text {. }}\right)}$ is the covariance between $y_{0 i 1}$ and $y_{0 i t_{i}}$ ). As sensitivity and specificity are estimated on different sets of patients, there is always zero within-study correlation between each pair of logit sensitivity and logit specificity estimates at a given threshold.

If two or more thresholds give identical results for sensitivity (or specificity) in a study, it is not then possible to estimate both thresholds in the same likelihood. This can be seen in the above $l_{i}(1)$ likelihood for the Al Ragib study, as $p_{1 i 3}$ is not identifiable due to no diseased patients having a test value falling between the second and third thresholds; thus the observed sensitivity is the same at $t_{i}=2$ and $t_{i}=3$. In this situation only one of the thresholds can be included in the likelihood, and so in Al Ragib, for example, one can exclude threshold $t_{i}=3$ and thus remove $p_{1 i 3}$ in $l_{i}(1)$. However the estimated logit value, its variance and covariances for the included threshold will be identical for the other excluded threshold(s). For this reason, the within-study correlation between thresholds with the same sensitivity (or specificity) will be 1, resulting in a singular covariance matrix. To make calculations possible, one can select the nearest-approximant positive definite matrix [33]; an easier to implement approach is to simply reduce the corrections slightly (e.g. map \pm 1 to \pm 0.95 ).

\section{Step 2: Multivariate meta-analysis}

The $y_{1 i_{i}}$ and $y_{0 i t_{i}}$ obtained from step 1 for all thresholds can now be synthesised simultaneously in a multivariate meta-analysis that accounts 
Citation: Riley RD, Takwoingi Y, Trikalinos T, Guha A, Biswas A, et al. (2014) Meta-Analysis of Test Accuracy Studies with Multiple and Missing Thresholds: A Multivariate-Normal Model. J Biomet Biostat 5: 196. doi:10.4172/2155-6180.1000196

for their within-study correlations. We now discuss two options for the multivariate model, one that treats the true logit sensitivities and logit specificities as separate (ordered) parameters, and one that assumes that they are monotonic functions of the (continuous) threshold.

\section{Option (i) - Multivariate meta-analysis with separate parameters for test performance at each threshold}

Let there be a total of $t=1$ to $T$ different thresholds across all studies (e.g. $T=23$ for the PCR data in Table 1), and let these be ordered in increasing value. Then in each study the $y_{1 i 1}, \ldots, y_{1 i t_{i}}$ and $y_{0 i 1}, \ldots, y_{0 i t_{i}}$ can be linked to one of these thresholds. If all studies report all thresholds, a multivariate-normal meta-analysis model assumes that:

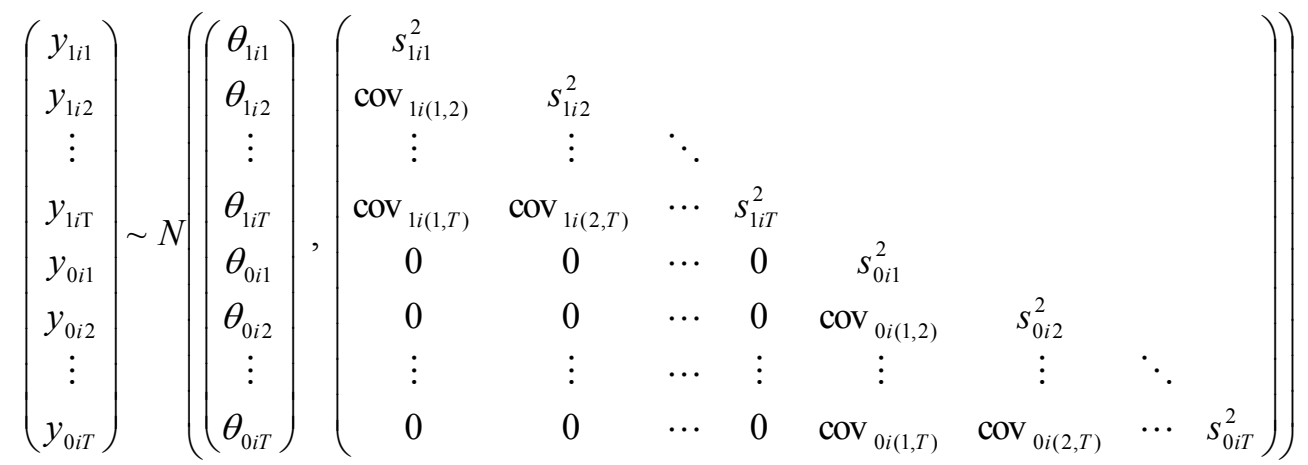

where

$$
\left(\begin{array}{c}
\theta_{1 i 1} \\
\theta_{1 i 2} \\
\vdots \\
\theta_{1 i T} \\
\theta_{0 i 1} \\
\theta_{0 i 2} \\
\vdots \\
\theta_{0 i T}
\end{array}\right) \sim N\left(\left(\begin{array}{c}
\beta_{11} \\
\beta_{12} \\
\vdots \\
\beta_{1 T} \\
\beta_{01} \\
\beta_{02} \\
\vdots \\
\beta_{0 T}
\end{array}\right), \Omega()\right.
$$

We might add ordering constraints so that mean logit sensitivities (mean logit specificities) decrease (increase) as the thresholds become higher: that is to constrain $\theta_{1 i j} \geq \theta_{1 i k}, \beta_{1 j} \geq \beta_{1 k} ; \theta_{0 i k} \geq \theta_{0 i j}, \beta_{0 k} \geq \beta_{0 j}$ for $j<k$.

In model (5) $\Omega$ is a $2 T$ by $2 T$ matrix representing the between-study variance-covariance matrix for the logit sensitivity and logit specificity values. The study estimates $\left(y_{1 i t_{i}}, y_{0 i t_{i}}\right)$, their variances $\left(s_{1 i t_{i}}^{2}, s_{0 i t_{i}}^{2}\right)$, and covariances (e.g. $\left.\operatorname{cov}_{1 i(1, t)}\right)$ are available from step one for each study. The variances and covariances are assumed known, which is a common meta-analysis assumption [34]. The model can easily accommodate missing thresholds in a study, assuming they are missing at random, just as described elsewhere for missing outcomes in a multivariate meta-analysis of multiple outcomes [11,27]. The model can be fitted using maximum likelihood estimation or restricted maximum likelihood (REML), for example in SAS Proc MIXED.

The $\beta_{1 t}$ and $\beta_{0 t}$ terms give the average logit sensitivity and average logit specificity at threshold $t$, respectively, and can be transformed back to give the summary sensitivity $\left(\exp \left(\beta_{1 t}\right) /\left(1+\exp \left(\beta_{1 t}\right)\right)\right.$ and the summary specificity $\left(\left(\exp \left(\beta_{0 t}\right) /\left(1+\exp \left(\beta_{0 t}\right)\right)\right.\right.$.

The number of parameters in the model is quadratic in $T$ : one has to estimate $2 T$ means, $2 T$ variances and $T(2 T-1)$ correlations. This makes fitting the model impractical for modest or large numbers of thresholds. For the PCR example (Table 1), this model would have 1127 parameters. Alternatively, one could impose structure to $\Omega$, to reduce the number of parameters to be estimated. We do not explore alternative structural specifications in this work. An additional complication is that one has to use convex optimization techniques to maximize the likelihood of the model, while respecting the inequality constraints. For these reasons, model (5) will often be impractical.

\section{Option (ii)-Multivariate meta-analysis assuming a functional relationship between test performance and threshold}

One can drastically reduce the number of parameters in the previous model by assuming that the true logit sensitivity (logit specificity) is a monotonic decreasing (increasing) function of the threshold. The simplest form is a linear relationship such that a one-unit increase in threshold value, $x$, leads to a constant change in logit sensitivity of $\gamma_{1}$, and a constant change in logit specificity of $\gamma_{0}$, with these changes assumed to be fixed across studies: 


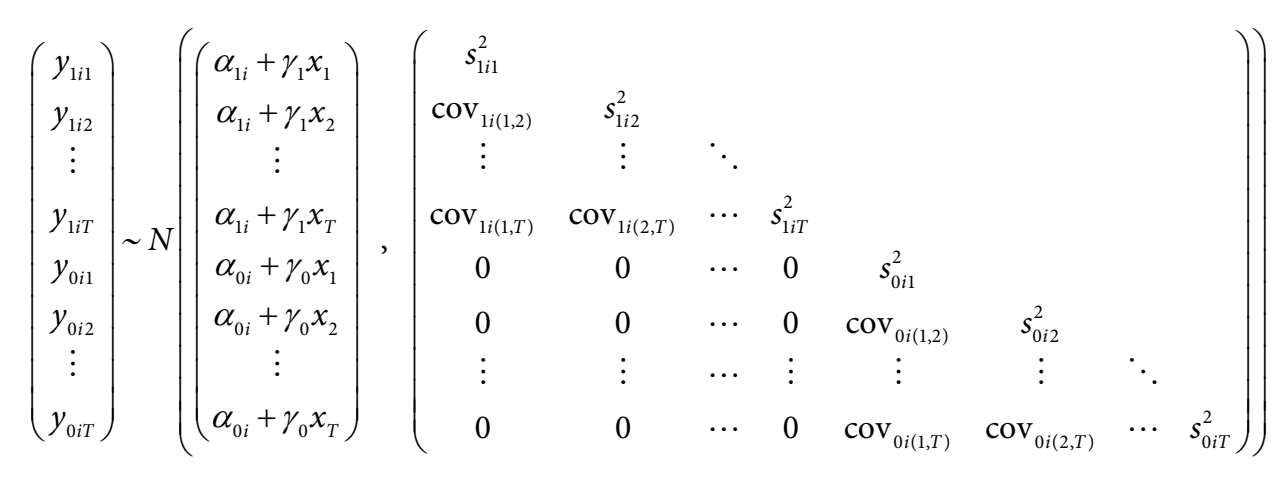

$$
\begin{aligned}
& \left(\begin{array}{c}
\alpha_{0 i} \\
\alpha_{1 i}
\end{array}\right) \sim N\left(\left(\begin{array}{c}
\alpha_{o} \\
\alpha_{1}
\end{array}\right),\left(\begin{array}{cc}
\tau_{\alpha_{0}}^{2} & \rho \tau_{\alpha_{0}} \tau_{\alpha_{1}} \\
\rho \tau_{\alpha_{0}} \tau_{\alpha_{1}} & \tau_{\alpha_{1}}^{2}
\end{array}\right)\right)
\end{aligned}
$$

where $x_{t}$ is the threshold value at the $t^{\text {th }}$ available threshold (where $t=1$ to $T$, and $T$ is the total number of different thresholds considered across all studies), and model (6) is subject to the constraints $\gamma_{1} \leq 0, \gamma_{0} \geq 0$.

In model (6) $\alpha_{1}$ and $\alpha_{0}$ are average intercepts, $\tau_{\alpha_{0}}^{2}$ and $\tau_{\alpha_{1}}^{2}$ are between-study variances and $\rho$ the between-study correlation. Model (6) is a multivariate mixed effects (random intercept/fixed slope) meta-regression with threshold as the only covariate. The effect of threshold on logit sensitivity and logit specificity is allowed to differ through different intercepts $\left(\alpha_{1 i}\right.$ and $\left.\alpha_{0 i}\right)$ and slopes $\left(\gamma_{1}\right.$ and $\left.\gamma_{0}\right)$. The summary ROC curve is therefore defined by $\alpha_{1}, \alpha_{0}, \gamma_{1}$ and $\gamma_{0}$. Model (6) has only 7 parameters instead of 1127 in model (5), at the cost of making strong assumptions about how test performance changes across thresholds. Extensions to model (6) may specify random slopes, but this may complicate estimation; indeed such an extension did not converge for the PCR example.

Fitting model (6) accounting for the constraints is straightforward (e.g. using REML in SAS Proc MIXED). As above one can accommodate missing thresholds in studies, assuming that they are missing at random, for example through a data-augmentation approach, where here the variances of missing threshold estimates are given a large value (e.g. 100000, and thus zero weighting) and zero covariances. Example SAS code for the data-augmentation and model fitting is available on request by the first author.

Summary estimates for logit sensitivity and specificity can be obtained after fitting model (6) for any threshold of choice (even those not reported in any of the studies) by:

$$
\begin{aligned}
& \text { logit sensitivity estimate at threshold } t=\hat{\alpha}_{1}+\hat{\gamma}_{1} x_{t} \\
& \text { logit specificity estimate at threshold } t=\hat{\alpha}_{0}+\hat{\gamma}_{0} x_{t}
\end{aligned}
$$

These can be transformed back to the sensitivity and specificity scale, and plotted together to produce a summary ROC curve to aid examination of test accuracy. Note that each point on the summary ROC curve relates to a known threshold value; this is not true for summary ROC curves derived from meta-analyses using a single threshold result from each study [3].

The linear relationships assumed in model (6) are a common assumption in meta-analysis of test accuracy studies producing summary ROC curves [14]. However other specifications can be examined, and the fit of the model investigated using statistics such as AIC and BIC. For example, below we extend model (6) to allow a cubic relationship:

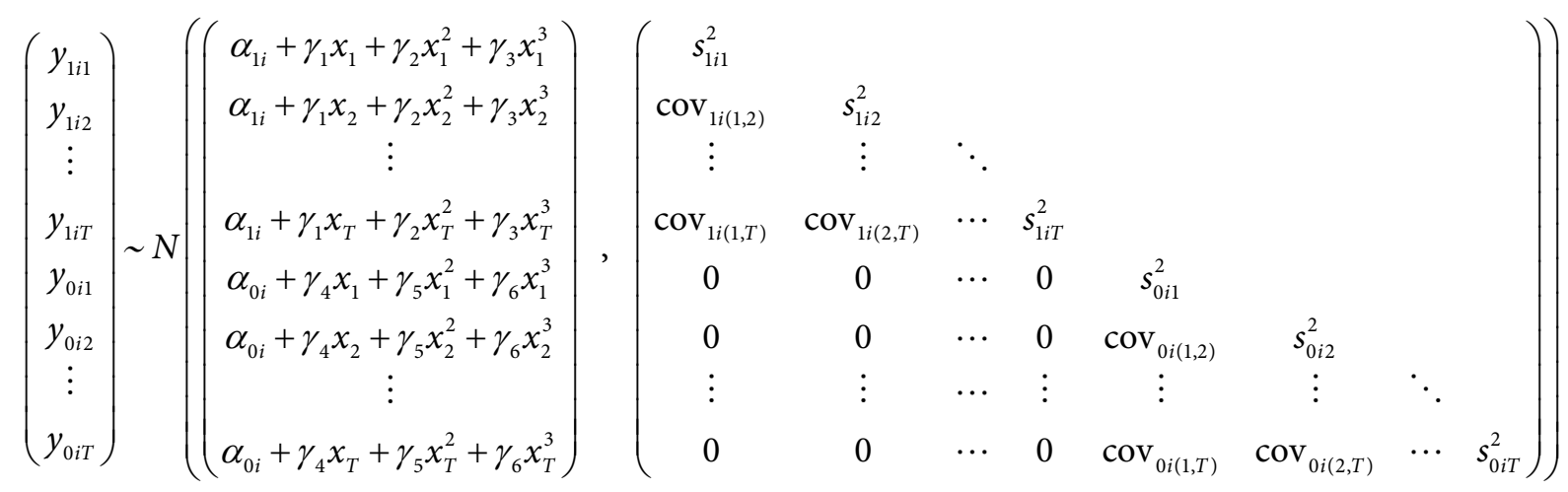

where the model is subject to the constraint that the polynomial $\gamma_{1} x_{t}+\gamma_{2} x_{t}^{2}+\gamma_{3} x_{t}^{3}$ is decreasing and the polynomial $\gamma_{4} x_{t}+\gamma_{5} x_{t}^{2}+\gamma_{6} x_{t}^{3}$ is increasing in the range of interest for the threshold values $x_{\mathrm{t}}$. The optimization of the constrained likelihood for model (9) may be difficult for some datasets, depending on the proportion of missing thresholds in studies. 
Citation: Riley RD, Takwoingi Y, Trikalinos T, Guha A, Biswas A, et al. (2014) Meta-Analysis of Test Accuracy Studies with Multiple and Missing Thresholds: A Multivariate-Normal Model. J Biomet Biostat 5: 196. doi:10.4172/2155-6180.1000196

\section{Results}

We now apply the methods described in Section 3 to the PCR data introduced in Section 2. Findings are described for each step of the methods outlined.

\section{Step 1: Individual study results}

In the PCR data, there are 23 different thresholds across studies $(\mathrm{T}=23)$. For each study we used SAS Proc NLMIXED to specify and then maximise the log of likelihoods (1) and (2) [29], to obtain estimates $y_{1 i t_{i}}$ and $y_{0 i t_{i}}$ for each available threshold in that study, and their withinstudy covariance matrix. Within-study correlations were generally very high and largest for pairs of thresholds closer together, emphasising the rationale for analysing all thresholds simultaneously. For example, in the Al Ragib study the correlation matrix for the logit sensitivity estimates was as follows:

\begin{tabular}{|c|c|c|c|c|c|}
\hline & \multicolumn{5}{|c|}{ Correlation matrix } \\
\hline threshold value, $x_{t}$ & $\mathbf{0 . 1 3}$ & $\mathbf{0 . 1 8}$ & $\mathbf{0 . 1 9}$ & $\mathbf{0 . 2}$ & 0.6655 \\
\hline $\mathbf{0 . 1 3}$ & 1 & 0.7928 & 0.7928 & 0.8394 & 0.7261 \\
\hline $\mathbf{0 . 1 8}$ & 0.7928 & 1.0000 & 1.0000 & 0.8394 & 0.7261 \\
\hline $\mathbf{0 . 1 9}$ & 0.7928 & 1.0000 & 1.0000 & 1.0000 & 0.8651 \\
\hline $\mathbf{0 . 2}$ & 0.6655 & 0.8394 & 0.8394 & 0.8651 & 1.0000 \\
\hline $\mathbf{0 . 4 9}$ & 0.5757 & 0.7261 & 0.7261 & \\
\hline
\end{tabular}

Notice here that the correlation matrix is singular (there is a correlation of +1 between logit sensitivities for thresholds 0.18 and 0.19 ). As mentioned in the methods, to allow estimation of meta-analysis models one can truncate correlations larger than 0.95 , to obtain an approximant invertible correlation matrix.

\section{Step 2: Meta-analysis results}

We transferred the $y_{1 i t}, y_{0 i t}$ and within-study covariance matrix to SAS for all studies and fitted the multivariate-normal meta-analysis model (6) using REML. (Model (5) was not fit because for $T=23$ thresholds it has many more parameters than the available data points). The model converged after about one hour, and the parameter estimates are shown in Table 2.

Summary ROC curve from model (6): The summary ROC curve from model (6) assumes a linear relationship between threshold value and logit sensitivity and logit specificity. The resultant curve in ROC space is shown in Figure 1, and the summary results for each threshold are shown in Table 3. Assuming that sensitivity and specificity are equally important (and thus that test accuracy is equally important for those with and without proteinuria), the threshold for which the sum of sensitivity and specificity is maximum is around a PCR value of 0.30 to 0.35 . PCR thresholds between 0.24 and 0.40 give summary sensitivity and specificity values that are both above $80 \%$, and a PCR value of 0.35 gives a similar summary sensitivity and specificity of 0.84 .

However, it is important to note the large estimated variances of the intercept terms in model (6), signalling large between-study heterogeneity in test accuracy at each threshold (and thus large heterogeneity in the ROC curve itself). The between-study standard deviation in logit sensitivity and logit specificity is 0.83 and 1.17 respectively: this indicates that, for example at a threshold with a summary sensitivity and specificity of $80 \%$, in a single population the true sensitivity may vary from 0.43 to 0.95 and the true specificity vary between 0.28 and 0.98 , approximately. The heterogeneity may be attributable to between-study differences in the enrolled populations or in the methods of measuring PCR, and indicates that further research is essential to establish the causes of heterogeneity. This is beyond the aims of this paper, but model (6) can be extended to examine additional study-level covariates that may explain the heterogeneity, at least in part. Note there was also a large between-study correlation of 0.94 between the model intercepts for sensitivity and specificity (Table 2), emphasising that studies with a higher true sensitivity are likely to have a higher true specificity.

\begin{tabular}{|c|c|c|}
\hline & estimate & \\
\hline Intercepts & & \\
\hline$\alpha_{0}$ & 0.851 & 0.354 \\
\hline$\alpha_{1}$ & 2.863 & 0.293 \\
\hline Slopes & & \\
\hline$\gamma_{0}$ & 2.309 & 0.307 \\
\hline$\gamma_{1}$ & -3.347 & 0.419 \\
\hline$\tau_{\alpha_{0}}^{2}$ & 1.369 & \\
\hline$\tau_{\alpha_{1}}^{2}$ & & \\
\hline$\rho$ & 0.687 & \\
\hline
\end{tabular}

Table 2: REML estimates of parameters in model (6) when applied to the PCR data. 
Citation: Riley RD, Takwoingi Y, Trikalinos T, Guha A, Biswas A, et al. (2014) Meta-Analysis of Test Accuracy Studies with Multiple and Missing Thresholds: A Multivariate-Normal Model. J Biomet Biostat 5: 196. doi:10.4172/2155-6180.1000196

Page 8 of 12

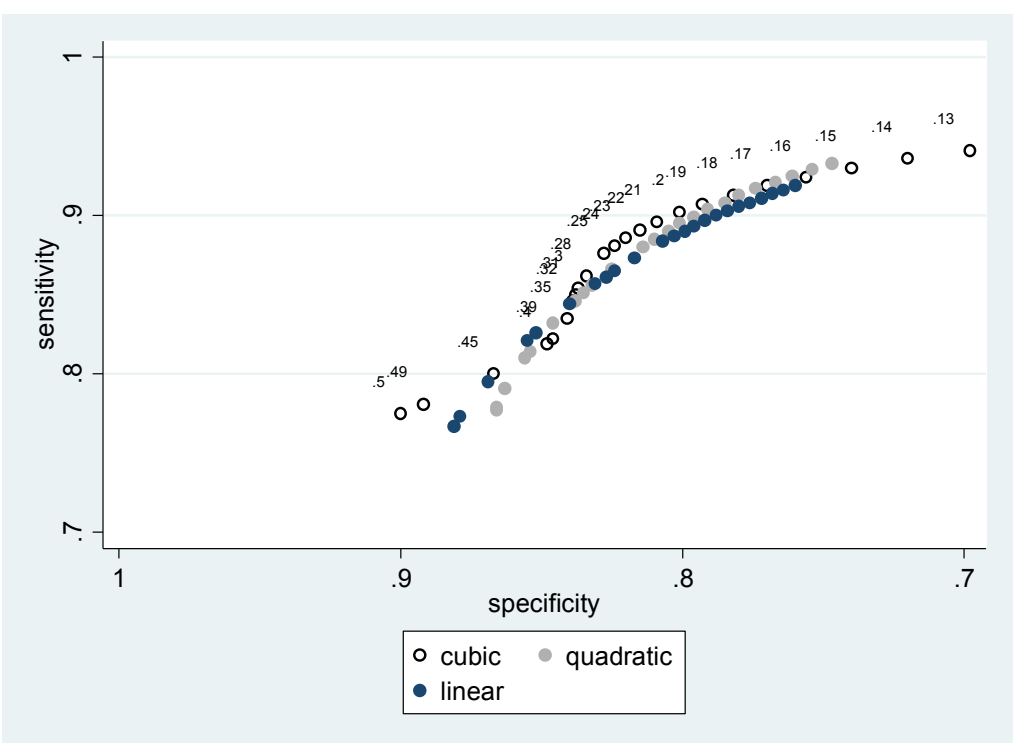

NB The number shown next to each hollow circle is the threshold value used to dichotomise PCR. Each set of circles are ordered in terms of threshold value, from a PCR of 0.13 for the circles farthest right, and a PCR of 0.50 for the circles farthest left.

Figure 1: Pair of summary sensitivity and specificity results for each threshold value, plotted in ROC space, for three different models: (i) model (6) with a linear trend, (ii) model (6) extended to include a quadratic trend, and (iii) model (6) with a cubic trend (i.e. model (9)).

\begin{tabular}{|c|c|c|c|c|c|c|c|}
\hline \multirow{4}{*}{$\begin{array}{c}\text { Threshold value, } x \\
0.13\end{array}$} & \multirow{4}{*}{$\begin{array}{l}\text { No. studies with this } \\
\text { threshold }\end{array}$} & \multicolumn{3}{|c|}{ Sensitivity } & \multicolumn{3}{|c|}{ Specificity } \\
\hline & & \multirow{3}{*}{$\begin{array}{c}\text { Summary estimate } \\
0.919\end{array}$} & \multicolumn{2}{|c|}{$95 \% \mathrm{Cl}$} & \multirow{3}{*}{$\begin{array}{c}\text { Summary estimate } \\
0.760\end{array}$} & \multicolumn{2}{|c|}{$95 \% \mathrm{Cl}$} \\
\hline & & & lower & upper & & lower & upper \\
\hline & & & 0.869 & 0.951 & & 0.615 & 0.862 \\
\hline 0.14 & 2 & 0.916 & 0.865 & 0.949 & 0.764 & 0.620 & 0.865 \\
\hline 0.15 & 5 & 0.914 & 0.862 & 0.947 & 0.768 & 0.626 & 0.868 \\
\hline 0.16 & 3 & 0.911 & 0.858 & 0.946 & 0.772 & 0.631 & 0.870 \\
\hline 0.17 & 3 & 0.908 & 0.854 & 0.944 & 0.776 & 0.637 & 0.873 \\
\hline 0.18 & 3 & 0.906 & 0.850 & 0.942 & 0.780 & 0.642 & 0.875 \\
\hline 0.19 & 4 & 0.903 & 0.846 & 0.940 & 0.784 & 0.648 & 0.878 \\
\hline 0.2 & 6 & 0.900 & 0.842 & 0.938 & 0.788 & 0.653 & 0.880 \\
\hline 0.21 & 3 & 0.897 & 0.837 & 0.936 & 0.792 & 0.658 & 0.883 \\
\hline 0.22 & 1 & 0.893 & 0.833 & 0.934 & 0.796 & 0.663 & 0.885 \\
\hline 0.23 & 1 & 0.890 & 0.828 & 0.932 & 0.799 & 0.668 & 0.887 \\
\hline 0.24 & 2 & 0.887 & 0.824 & 0.929 & 0.803 & 0.673 & 0.890 \\
\hline 0.25 & 2 & 0.884 & 0.819 & 0.927 & 0.807 & 0.679 & 0.892 \\
\hline 0.28 & 2 & 0.873 & 0.804 & 0.920 & 0.817 & 0.693 & 0.898 \\
\hline 0.3 & 4 & 0.865 & 0.793 & 0.915 & 0.824 & 0.703 & 0.903 \\
\hline 0.31 & 1 & 0.861 & 0.788 & 0.912 & 0.827 & 0.708 & 0.905 \\
\hline 0.32 & 1 & 0.857 & 0.782 & 0.909 & 0.831 & 0.712 & 0.907 \\
\hline 0.35 & 1 & 0.844 & 0.764 & 0.901 & 0.840 & 0.726 & 0.912 \\
\hline 0.39 & 2 & 0.826 & 0.739 & 0.888 & 0.852 & 0.743 & 0.920 \\
\hline 0.4 & 2 & 0.821 & 0.732 & 0.885 & 0.855 & 0.748 & 0.922 \\
\hline 0.45 & 1 & 0.795 & 0.697 & 0.868 & 0.869 & 0.768 & 0.930 \\
\hline 0.49 & 1 & 0.773 & 0.666 & 0.853 & 0.879 & 0.783 & 0.936 \\
\hline 0.5 & 2 & 0.767 & 0.658 & 0.849 & 0.881 & 0.787 & 0.937 \\
\hline
\end{tabular}

Table 3: Summary results from REML estimation of meta-analysis model (6) (linear relationship).

Summary ROC curve from non-linear extensions to model (6): As mentioned, model (6) can also be extended to allow non-linear relationships between threshold value and logit sensitivity and logit specificity. We considered quadratic and cubic extensions to model (6): the cubic extension is as written in model (9). The summary ROC curves are shown in Figure 1, and the cubic model was the better fit (AIC: 5548 for cubic, 5591 for quadratic, 5605 for linear). Other fractional polynomial terms were also considered, but did not improve model fit. The cubic model stretches out the summary ROC curve compared to linear and quadratic models, and in particular gives lower summary specificity estimates at the lower PCR 
(a)

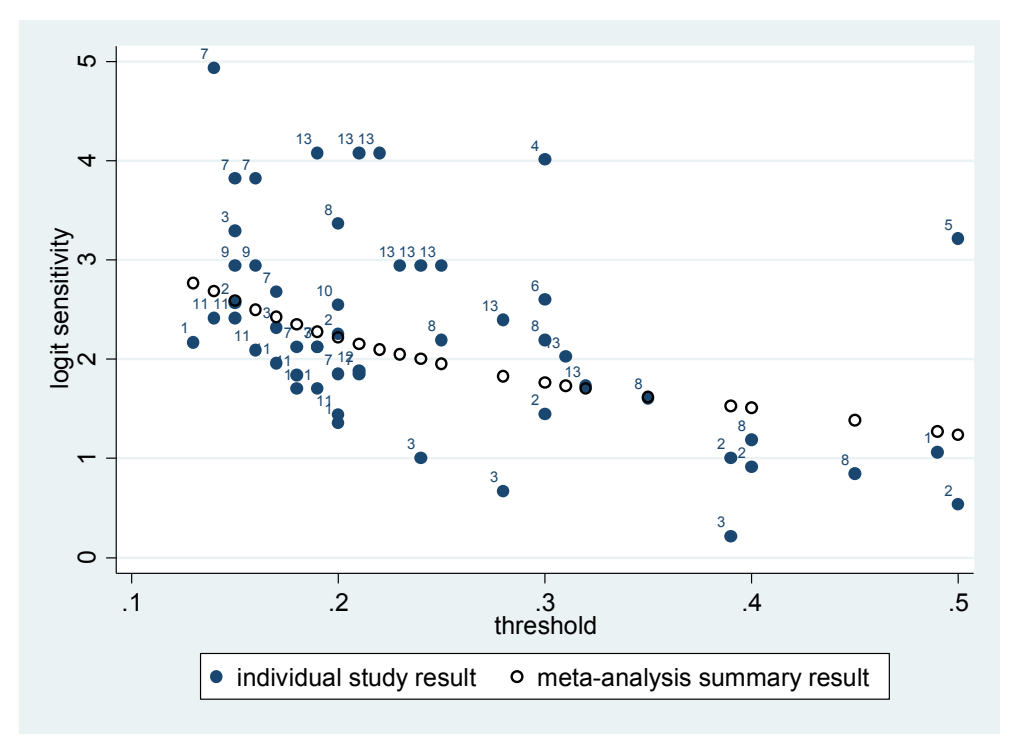

(b)

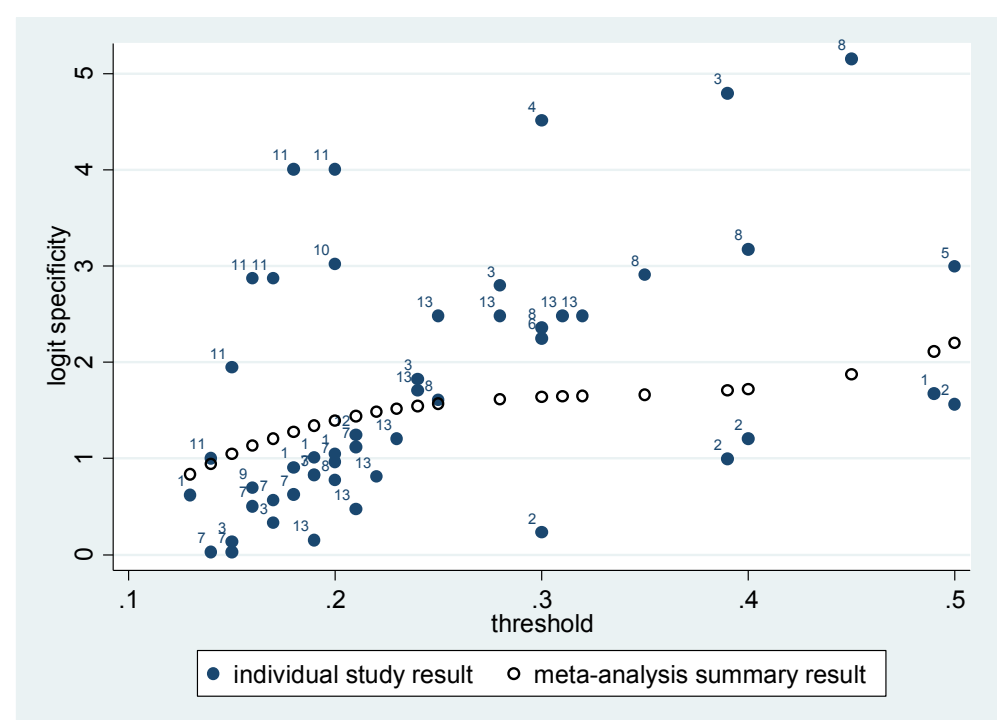

Numbers next to the points denote study ID number. In each figure, the hollow circles are ordered in terms of threshold value, from a PCR of 0.13 for the circles farthest right, and a PCR of 0.50 for the circles farthest left.

Figure 2: Fit of meta-analysis model (9) with a cubic summary relationship between threshold value and (a) the study logit sensitivity estimates, and (b) the study logit specificity estimates.

thresholds. The summary results from the cubic model are shown in Table 4, and the fit of the cubic model is shown in Figure 2. As before, when assuming sensitivity and specificity are equally important, the best threshold appears to be around 0.30 to 0.35 .

Comparison to a separate analysis at each threshold: When presented with multiple thresholds per study, researchers often do a separate meta-analysis for each threshold. In comparison to a multivariate meta-analysis, this loses information and may make summary results hard to interpret. For example, consider a PCR threshold of 0.23: this was only considered by one study ('Yamasmit', Table 1). Based on this single study, the summary result for sensitivity is 0.95 . A threshold of 0.13 is also considered by just one study ('Al Ragib', Table 1 ), and the summary sensitivity is 0.90 . Therefore the higher threshold of 0.23 is giving a higher sensitivity estimate; this is clearly not helpful, as the summary sensitivity should decrease as threshold increases. In contrast, the multivariate model (6) (and its extensions such as model (9)) constrain results, so that the summary sensitivity estimates are now more appropriate, being 0.92 and 0.89 for thresholds 0.13 and 0.23 respectively in model (6) (Table 3).

Summary results for entirely missing thresholds: Finally, model (6) and model (9) produce summary results for each threshold reported in the literature, but also allow summary results for other unreported thresholds of interest. For example, consider a PCR threshold value of 0.29 . 
Citation: Riley RD, Takwoingi Y, Trikalinos T, Guha A, Biswas A, et al. (2014) Meta-Analysis of Test Accuracy Studies with Multiple and Missing Thresholds: A Multivariate-Normal Model. J Biomet Biostat 5: 196. doi:10.4172/2155-6180.1000196

Page 10 of 12

\begin{tabular}{|c|c|c|c|c|c|c|c|}
\hline \multirow{3}{*}{$\begin{array}{c}\text { Threshold value, } x \\
0.13\end{array}$} & \multirow{3}{*}{$\begin{array}{c}\text { No. studies with this } \\
\text { threshold } \\
1\end{array}$} & \multicolumn{3}{|c|}{ Sensitivity } & \multicolumn{3}{|c|}{ Specificity } \\
\hline & & \multirow{2}{*}{$\begin{array}{c}\text { Summary estimate } \\
0.941\end{array}$} & \multicolumn{2}{|c|}{$95 \% \mathrm{Cl}$} & \multirow{2}{*}{$\begin{array}{c}\text { Summary estimate } \\
0.698\end{array}$} & $95 \% \mathrm{Cl}$ & upper \\
\hline & & & 0.896 & 0.967 & & 0.544 & 0.818 \\
\hline 0.14 & 2 & 0.936 & 0.889 & 0.963 & 0.720 & 0.572 & 0.832 \\
\hline 0.15 & 5 & 0.930 & 0.882 & 0.959 & 0.740 & 0.597 & 0.845 \\
\hline 0.16 & 3 & 0.924 & 0.875 & 0.955 & 0.756 & 0.618 & 0.856 \\
\hline 0.17 & 3 & 0.919 & 0.867 & 0.951 & 0.770 & 0.637 & 0.865 \\
\hline 0.18 & 3 & 0.913 & 0.859 & 0.948 & 0.782 & 0.653 & 0.873 \\
\hline 0.19 & 4 & 0.907 & 0.851 & 0.944 & 0.793 & 0.666 & 0.880 \\
\hline 0.2 & 6 & 0.902 & 0.843 & 0.940 & 0.801 & 0.678 & 0.885 \\
\hline 0.21 & 3 & 0.896 & 0.835 & 0.937 & 0.809 & 0.688 & 0.890 \\
\hline 0.22 & 1 & 0.891 & 0.827 & 0.933 & 0.815 & 0.697 & 0.894 \\
\hline 0.23 & 1 & 0.886 & 0.820 & 0.930 & 0.820 & 0.704 & 0.897 \\
\hline 0.24 & 2 & 0.881 & 0.812 & 0.926 & 0.824 & 0.709 & 0.900 \\
\hline 0.25 & 2 & 0.876 & 0.805 & 0.923 & 0.828 & 0.714 & 0.902 \\
\hline 0.28 & 2 & 0.862 & 0.786 & 0.914 & 0.834 & 0.724 & 0.907 \\
\hline 0.3 & 4 & 0.854 & 0.774 & 0.909 & 0.837 & 0.727 & 0.908 \\
\hline 0.31 & 1 & 0.850 & 0.768 & 0.906 & 0.838 & 0.729 & 0.909 \\
\hline 0.32 & 1 & 0.846 & 0.763 & 0.904 & 0.839 & 0.730 & 0.909 \\
\hline 0.35 & 1 & 0.835 & 0.748 & 0.897 & 0.841 & 0.733 & 0.910 \\
\hline 0.39 & 2 & 0.822 & 0.729 & 0.888 & 0.846 & 0.740 & 0.914 \\
\hline 0.4 & 2 & 0.819 & 0.724 & 0.886 & 0.848 & 0.743 & 0.915 \\
\hline 0.45 & 1 & 0.800 & 0.699 & 0.874 & 0.867 & 0.770 & 0.927 \\
\hline 0.49 & 1 & 0.781 & 0.674 & 0.860 & 0.892 & 0.806 & 0.943 \\
\hline 0.5 & 2 & 0.775 & 0.665 & 0.857 & 0.900 & 0.816 & 0.948 \\
\hline
\end{tabular}

Table 4: Summary results from REML estimation of meta-analysis model (9) (cubic relationship).

Results for this threshold were not reported in any of the studies, but following model (9) the summary logit sensitivity for the threshold can be obtained by $\hat{\alpha}_{1}+\hat{\gamma}_{1} 0.29+\hat{\gamma}_{2} 0.29^{2}+\hat{\gamma}_{3} 0.29^{3}$ and the summary specificity by $\hat{\alpha}_{0}+\hat{\gamma}_{4} 0.29+\hat{\gamma}_{5} 0.29^{2}+\hat{\gamma}_{6} 0.29^{3}$. This gives a summary logit sensitivity of 0.858 and a summary specificity of 0.836 , constrained to fall between the summary results for the reported thresholds of 0.28 and 0.30 (Table 4).

\section{Discussion}

Many meta-analysis articles have considered how to combine multiple test accuracy studies each presenting a single threshold, even if the threshold used in each study differs [1-8]. However, there have been only a few papers considering how to combine studies when some report results for multiple thresholds [13-15]. We have considered a multivariate-normal approach for this situation, and shown its potential benefits and limitations through an applied example. The approach allows all studies to be included, regardless of how many thresholds they present, and incorporates all reported thresholds even those just reported in a single study. The example shows that model (6) and subsequent extensions produce the more coherent results, constrained to be ordered across thresholds. It produces summary estimates of sensitivity and specificity for each threshold; estimates of between-study heterogeneity; and an admissible summary ROC curve, whilst always accounting for within-study correlation of results for multiple thresholds from the same study. As a consequence, the methods utilise more information than an approach that analyses each threshold independently, and thereby it reduces the impact of missing thresholds in the studies available. Summary results are produced for each threshold reported in the literature, and can even be derived for unreported thresholds that are of interest.

We note that our models (6) and (9) imply a specific distribution for test results in people with and without the condition of interest in each study. The same is true for any model that posits functionals between the true sensitivities (specificities) and the threshold (e.g., Hamza et al. [14], or Putter et al.[15]). If the study-level data do not fit the implied distribution, predictions of summary sensitivity and specificity at various thresholds would be biased. Model (5) does not posit such functionals, and only assumes that true (logit) sensitivities and specificities at adjacent thresholds are ordered. However, relaxing the assumption comes at a large cost: for $T=23$ thresholds, model (5) has 1127 parameters, instead of only 7 in model (6). In situations with a smaller set of thresholds, for example $T=2,3$ or 4, model (5) may be more feasible. Indeed, it may be preferable as with only a few thresholds models (6) and (9) may not converge: the fewer the data points the harder it is to estimate a functional relationship between the true sensitivities (specificities) and the threshold. In situation with only a few studies none of models (5), (6) or (9) maybe achievable, especially when the number of thresholds is large. The issue would be resolved if individual participant data (IPD) were available for each study, as then all thresholds are available for all studies. This allows the functional relationship to be estimated separately in each study based on the discrete (exact) likelihood [14], and the parameter estimates of the function then pooled across studies, similar to the IPD meta-analysis of longitudinal data [35].

Multivariate meta-analysis models are increasingly used to synthesise multiple correlated outcomes in meta-analysis [11], to improve the efficiency of summary estimates by borrowing strength across outcomes and thereby reducing the impact of missing data [12,36]. In this work we used the multivariate normal approximation to the multinomial distribution to model the observed data in each study. This approximation may be unsatisfactory for small sample sizes or for proportions near 0 or $1[2,37,38]$. In many applied examples, this limitation may not be a big problem. 
The advantage is that it is very easy to fit within standard software. Alternative models use discrete likelihoods for the observed data (e.g. see Hamza et al. [14]). However, they are more difficult to fit with random-effects and missing data, and often they do not converge [15]. One could simplify matters by meta-analysing each threshold separately and excluding studies that do not report that threshold: this would allow the exact binomial distribution and removes studies with missing data [2]. However, taking each threshold separately does not ensure summary results are ordered across thresholds, especially if studies have missing thresholds as in the PCR data. Therefore, although approximate, the proposed multivariatenormal model remains appealing, and may be considered in place of models using discrete likelihoods, for example when they fail to converge (as they did for the PCR data). We assumed the logit transformed sensitivity and specificity estimates follow a multivariate normal distribution in each study, but note log-log or probit transformations could also be considered if more suitable [28].

Our methods also allow studies providing IPD to be combined with studies only providing two by two tables for a partial set of thresholds. Essentially two by two tables for a full set of thresholds can be derived in IPD studies, which can then be combined with the other studies using the methods proposed. IPD would also allow the distribution of continuous test values for diseased and non-diseased patients to be modelled in each study; these distribution might then be synthesised across studies to form summary distributions, from which the summary ROC could easily be derived. With only aggregate data from non-IPD studies it is harder to derive the distributions of the original data, but they might be approximately calculable and we are considering this in further research.

In some situations sensitivity analyses may be useful, where the multivariate model is re-fitted to a subset of available studies. For example, if some studies are classed at a high risk of bias then one might investigate if summary meta-analysis conclusions are sensitive to their inclusion. Similarly if studies that use a different reference standard or method of test measurement may be omitted for sensitivity analysis. This was not considered in the original PCR meta-analysis [10], and so was not evaluated here.

Finally, we note that model (6) can be extended to compare multiple tests. Additional covariates can be included in the regression part of the model to distinguish each test, and if some studies consider two or more tests, the within-study correlation in the multiple test results can be accounted for in the same manner as described. The shape of the summary ROC curve may be forced to be the same for each test, or allowed to differ. Either way, one can compare the summary ROC curves obtained for the tests, to determine which test is more accurate [39].

\section{Conclusion}

We have presented a multivariate-normal method to deal with multiple and missing thresholds across studies in a meta-analysis. The method offers an approximate alternative when more exact methods experience computational problems or require complete data. By utilising correlation between thresholds the method limits the impact of missing threshold results from primary studies and ensures a summary ROC curve that, unlike a separate meta-analysis at each threshold, is constrained to be ordered across thresholds.

\section{Acknowledgments}

RDR and JJD were supported by funding from an MRC Methodology Research Grant in Multivariate Meta-analysis (grant reference number: MR/J013595/1). RKM is funded by an NIHR Clinical Lectureship.

\section{References}

1. Reitsma JB, Glas AS, Rutjes AW, Scholten RJ, Bossuyt PM, et al. (2005) Bivariate analysis of sensitivity and specificity produces informative summary measures in diagnostic reviews. J Clin Epidemiol 58: 982-990.

2. Chu H, Cole SR (2006) Bivariate meta-analysis of sensitivity and specificity with sparse data: a generalized linear mixed model approach. J Clin Epidemiol 59: 13311332.

3. Rutter CM, Gatsonis CA (2001) A hierarchical regression approach to meta-analysis of diagnostic test accuracy evaluations. Stat Med 20: 2865-2884

4. Harbord RM, Deeks JJ, Egger M, Whiting P, Sterne JA (2007) A unification of models for meta-analysis of diagnostic accuracy studies. Biostatistics 8: 239-251.

5. Moses LE, Shapiro D, Littenberg B (1993) Combining independent studies of a diagnostic test into a summary ROC curve: data-analytic approaches and some additional considerations. Stat Med 12: 1293-1316.

6. Littenberg B, Moses LE (1993) Estimating diagnostic accuracy from multiple conflicting reports: a new meta-analytic method. Med Decis Making $13: 313-321$.

7. Macaskill P (2004) Empirical Bayes estimates generated in a hierarchical summary ROC analysis agreed closely with those of a full Bayesian analysis. J Clin Epidemiol 57: 925-932.

8. Deeks JJ (2001) Systematic reviews in health care: Systematic reviews of evaluations of diagnostic and screening tests. BMJ $323: 157-162$.

9. Hewitt C, Gilbody S, Brealey S, Paulden M, Palmer S, et al. (2009) Methods to identify postnatal depression in primary care: an integrated evidence synthesis and value of information analysis. Health Technol Assess 13: 147-230.

10. Morris RK, Riley RD, Doug M, Deeks JJ, Kilby MD (2012) Diagnostic accuracy of spot urinary protein and albumin to creatinine ratios for detection of significant proteinuria or adverse pregnancy outcome in patients with suspected pre-eclampsia: systematic review and meta-analysis. BMJ 345 : e4342.

11. Jackson D, Riley R, White IR (2011) Multivariate meta-analysis: Potential and promise. Stat Med

12. Riley RD (2009) Multivariate meta-analysis: the effect of ignoring within-study correlation. JRSS Series A 172: $789-811$.

13. Dukic V, Gatsonis C (2003) Meta-analysis of diagnostic test accuracy assessment studies with varying number of thresholds. Biometrics 59: $936-946$.

14. Hamza TH, Arends LR, van Houwelingen HC, Stijnen T (2009) Multivariate random effects meta-analysis of diagnostic tests with multiple thresholds. BMC Med Res Methodol 9: 73.

15. Putter H, Fiocco M, Stijnen T (2010) Meta-analysis of diagnostic test accuracy studies with multiple thresholds using survival methods. Biom J 52: $95-110$.

16. Irwig L, Tosteson AN, Gatsonis C, Lau J, Colditz G, et al. (1994) Guidelines for meta-analyses evaluating diagnostic tests. Ann Intern Med 120: $667-676$. 
Citation: Riley RD, Takwoingi Y, Trikalinos T, Guha A, Biswas A, et al. (2014) Meta-Analysis of Test Accuracy Studies with Multiple and Missing Thresholds: A Multivariate-Normal Model. J Biomet Biostat 5: 196. doi:10.4172/2155-6180.1000196

Page 12 of 12

17. Tosteson AN, Begg CB (1988) A general regression methodology for ROC curve estimation. Med Decis Making 8: $204-215$.

18. Kester AD, Buntinx F (2000) Meta-analysis of ROC curves. Med Decis Making 20: 430-439.

19. Poon WY (2004) A latent normal distribution model for analysing ordinal responses with applications in meta-analysis. Stat Med 23: $2155-2172$.

20. Bipat S, Zwinderman AH, Bossuyt PM, Stoker J (2007) Multivariate random-effects approach: for meta-analysis of cancer staging studies. Acad Radiol 14: $974-984$.

21. CEMACH (2005) Saving Mothers' Lives: reviewing maternal deaths to make motherhood safer-2003-2005. The Seventh Report on Confidential Enquiries into Maternal Deaths in the United Kingdom. The Confidential Enquiry into Maternal and Child Health (CEMACH).

22. Montan S, Liedholm H, Lingman G, Marsál K, Sjöberg NO, et al. (1987) Fetal and uteroplacental haemodynamics during short-term atenolol treatment of hypertension in pregnancy. Br J Obstet Gynaecol 94: 312-317.

23. Khan KS, Wojdyla D, Say L, Gülmezoglu AM, Van Look PF (2006) WHO analysis of causes of maternal death: a systematic review. Lancet 367: 1066-1074

24. WHO (1988) Geographic variation in the incidence of hypertension in pregnancy. World Health Organization International Collaborative Study of Hypertensive Disorders of Pregnancy. Am J Obstet Gynecol 158: 80-83.

25. Brown MA, Lindheimer MD, de Swiet M, Van Assche A, Moutquin JM (2001) The classification and diagnosis of the hypertensive disorders of pregnancy: statement from the International Society for the Study of Hypertension in Pregnancy (ISSHP). Hypertens Pregnancy 20: IX-XIV.

26. White IR (2009) Multivariate meta-analysis. The Stata Journal 9: 40-56.

27. Riley RD, Abrams KR, Lambert PC, Sutton AJ, Thompson JR (2007) An evaluation of bivariate random-effects meta-analysis for the joint synthesis of two correlated outcomes. Stat Med 26: 78-97.

28. Chu H, Guo H, Zhou Y (2010) Bivariate random effects meta-analysis of diagnostic studies using generalized linear mixed models. Med Decis Making $30: 499-508$.

29. SAS Institute Inc (1999) SAS Institute Inc. PROC NLMIXED. Cary, NC: SAS Institute Inc.

30. Gould W, Pitblado J, Sribney W (2003) Maximum Likelihood Estimation with Stata, (2ndedn), Stata Press.

31. Billingsley P (1986) Probability and Measure. (2ndedn), John Wiley \& Sons Inc., New York.

32. Cox C (1998) Delta Method. In: Armitage P, Colton T, editors. Encyclopedia of Biostatistics. John Wiley, New York, pp. $1125-1127$.

33. Higham N (2002) Computing the nearest correlation matrix - a problem from finance. IMA Journal of Numerical Analysis 22: $329-343$.

34. Higgins JP, Thompson SG, Spiegelhalter DJ (2009) A re-evaluation of random-effects meta-analysis. J R Stat Soc Ser A Stat Soc 172: $137-159$.

35. Jones AP, Riley RD, Williamson PR, Whitehead A (2009) Meta-analysis of individual patient data versus aggregate data from longitudinal clinical trials. Clin Trials 6 : 16-27.

36. Kirkham JJ, Riley RD, Williamson PR (2012) A multivariate meta-analysis approach for reducing the impact of outcome reporting bias in systematic reviews. Stat Med 31: 2179-2195.

37. Hamza TH, van Houwelingen HC, Stijnen T (2008) The binomial distribution of meta-analysis was preferred to model within-study variability. J Clin Epidemiol 61: $41-51$.

38. Stijnen T, Hamza TH, Ozdemir P (2010) Random effects meta-analysis of event outcome in the framework of the generalized linear mixed model with applications in sparse data. Stat Med 29: 3046-3067.

39. Takwoingi Y, Leeflang MM, Deeks JJ (2013) Empirical evidence of the importance of comparative studies of diagnostic test accuracy. Ann Intern Med 158: $544-554$. 